\title{
Várices ectópicas en yeyuno proximal asociadas a sangrado recurrente en paciente sin cirrosis hepática
}

\section{Ectopic Varices in the Proximal Jejunum Associated with Recurrent Bleeding in a Patient without Cirrhosis of the Liver}

Mauricio Melo Peñaloza, MD, ${ }^{1}$ María Teresa Galiano de Sánchez, MD. ${ }^{2}$

\author{
' Gastroenterólogo, Clínica Martha. Villavicencio \\ (Meta), Colombia \\ 2 Gastroenteróloga, Clínica de Marly. Bogotá, \\ Colombia \\ Fecha recibido: $\quad 03-02-14$ \\ Fecha aceptado: $05-11-14$
}

\begin{abstract}
Resumen
Las várices ectópicas (VE) en yeyuno son alteraciones vasculares infrecuentes, asociadas en mayor medida a la hipertensión portal (5\%). Cuando se presenta sin esta asociación son aún menos frecuentes.

Presentamos un caso de sangrado crónico intestinal de varios años, sin hipertensión portal proveniente de várices localizadas en yeyuno proximal, su curso clínico, los elementos de diagnóstico empleados y terapia quirúrgica.
\end{abstract}

Palabras clave

Hipertensión portal, várices yeyunales, sangrado intestinal bajo oculto u oscuro.

\begin{abstract}
Ectopic varices (EV) in the jejunum are a rare vascular disorder which is associated with portal hypertension $(5 \%)$. It is even rarer when it occurs without associated portal hypertension.

We report a case of a patient without portal hypertension who had had chronic intestinal bleeding from varices located in the proximal jejunum for several years. We report the patient's clinical development, elements used for diagnosis and the surgical treatment.
\end{abstract}

\section{Keywords}

Portal hypertension, jejunal varices, occult and obscure intestinal bleeding.

\section{INTRODUCCIÓN}

Se define como sangrado intestinal oscuro al sangrado que persiste o recurre sin obvia etiología luego de practicar endoscopia digestiva alta y colonoscopia y puede ser oscuro evidente o manifiesto cuando el paciente y el clínico evidencian el sangrado u oscuro oculto que no es precisable y los exámenes paraclínicos lo determinan $(1,15)$.

El sangrado intestinal bajo afecta predominantemente a poblaciones con promedio de edad mayor a 65 años. La incidencia anual en la tercera década es de 1 por 100.000 y puede llegar a 200 por 100.000 en personas de la novena década. Algunos estudios muestran que 70\% de los afectados tienen al menos una enfermedad coexistente $(3,9,14)$.

Uno de los exámenes de laboratorio que puede ayudar en la diferenciación del sangrado es el BUN (nitrógeno ureico en sangre), porque indicaría presencia de sangrado y la razón $\mathrm{BUN} /$ creatinina $>33$ podría indicar que el sangrado es de origen alto o de intestino delgado pero no de colon, en pacientes sin antecedente de enfermedad renal crónica o aguda asociada $(9,10)$.

Aproximadamente 5\% del sangrado intestinal ocurre entre el ligamento de Treitz y la válvula ileocecal. Las lesiones vasculares corresponden a $40 \%$ de las causas de sangrado y 
de estas las angiectasias o angiodisplasias son el origen más común en pacientes ancianos; menos frecuentes como causa vascular están la fístula aortoentérica, la hemobilia, hemosuccus pancreático y várices del intestino delgado; otras lesiones diferentes a las vasculares son daños por antiinflamatorios no esteroideos y tumores $(1-8,14,15)$.

Con la llegada de nuevas técnicas como la cápsula endoscópica y la enteroscopia, la tendencia es a redefinir el sangrado intestinal general y el sangrado intestinal oscuro en tres categorías: alto, medio y bajo en vez de las dos tradicionales alto y bajo.

El sangrado con origen por encima de la ampolla de váter es el alto, el sangrado con origen entre la ampolla de váter y el íleon que es mejor investigado por cápsula endoscópica o enteroscopia (de empuje y la asistida con doble balón) es definido como sangrado medio y el sangrado con origen en colon es definido como bajo y es evaluado por la colonoscopia $(1,2)$.

Si no se identifican lesiones pero el sangrado es activo se podría recurrir al escáner por radionúclidos, a la angiografía, o a la cirugía; en caso de que no se encuentren lesiones y no se evidencie sangrado, el siguiente paso es la utilización, como método diagnóstico, de la enteroscopia o la videocápsula endoscópica (figura 1) $(3,6-8,12)$.

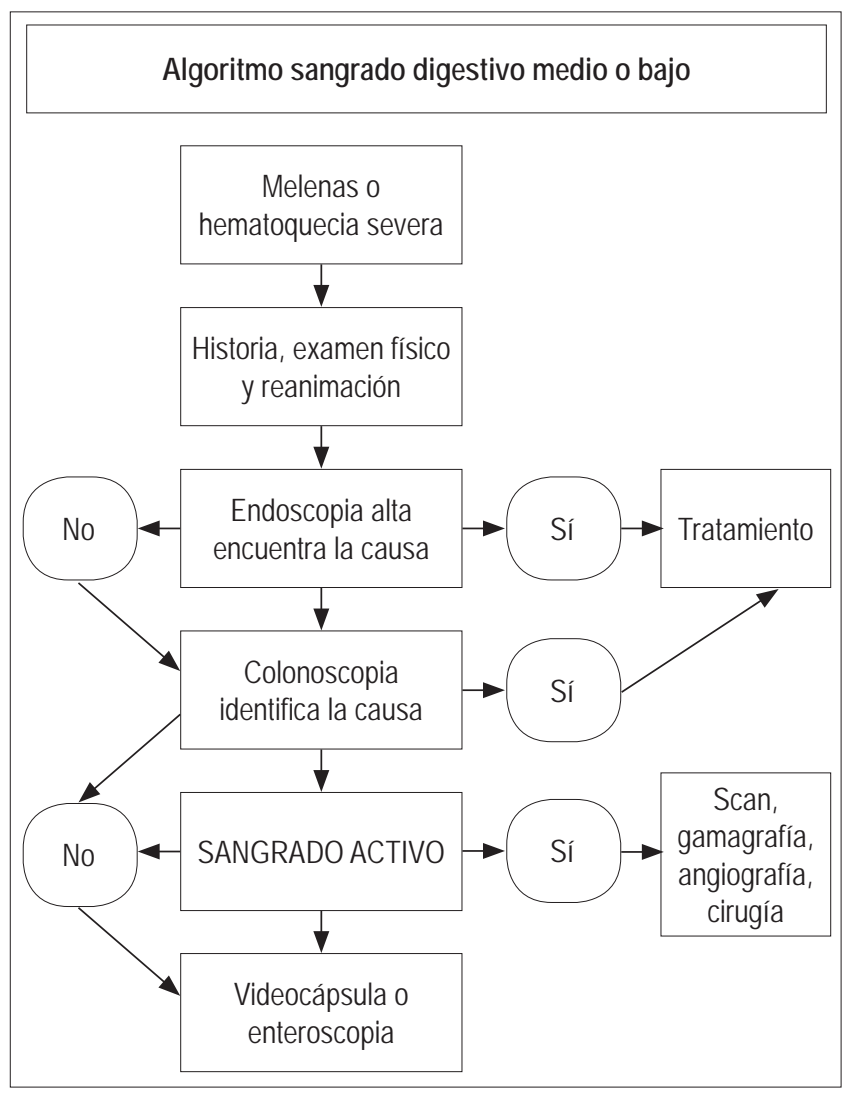

Figura 1. Diagrama de seguimiento del sangrado digestivo bajo oculto u oscuro. Modificada de referencia 6.
La enteroscopia tiene en la actualidad dos formas: la estereoscopia de empuje y la asistida con doble balón. La diferencia entre los dos métodos está en la longitud alcanzada en el intestino delgado y por tanto la cantidad de veces que se llega al diagnóstico. En la enteroscopia de empuje se llega a alcanzar una longitud de hasta $80 \mathrm{~cm}$ en yeyuno y en la enteroscopiaasistida con doble balón hasta $230 \mathrm{~cm}$, longitud que hace que se puedan encontrar un mayor número de lesiones $(11,12)$.

La enteroscopia intraoperatoria se considera como último recurso cuando los otros métodos no hayan podido esclarecer la causa del sangrado, ya que si bien los resultados en cuanto a diagnóstico son altos (79\%), se ha asociado a complicaciones posprocedimiento (adherencias, perforación, infección, hernias) que va de 5 a $20 \%$, especialmente en pacientes con comorbilidades $(6,13)$.

\section{CASO CLÍNICO}

Paciente femenina de 52 años, raza negra, hipertensa y diabética. La primera consulta atribuible a su problema actual se presentó el 15 de enero de 2009, y manifestaba múltiples deposiciones diarreicas con moco, asociado a dolor abdominal de tipo cólico, síntomas manejados de manera sintomática. Traía informe de colonoscopia de 2003 hasta colon ascendente que es normal.

Consulta en septiembre de ese año, por síntomas de dolor torácico y anemia $(\mathrm{Hb} 4,2 \mathrm{Gr} / \mathrm{dl})$ se le transfundieron glóbulos rojos, se descartó enfermedad coronaria y no se hizo estudio gastroenterológico.

Un mes después consulta por melenas en múltiples ocasiones, es tratada con transfusiones de glóbulos rojos, y dada de alta.

$\mathrm{Al}$ año siguiente en dos controles de consulta externa se encontró $\mathrm{Hb} 9,1 \mathrm{Gr} / \mathrm{dl}$ y valores de BUN de 60 y $110 \mathrm{mg} /$ $\mathrm{dl}(\mathrm{VN} 6$ a $20 \mathrm{mg} / \mathrm{dl})$.

A mediados de 2010 se encontró $\mathrm{Hb} 7,2 \mathrm{Gr} / \mathrm{dl}$ y es nuevamente transfundida con glóbulos rojos. Se practicó endoscopia alta con reporte de gastritis antral y colonoscopia informada hasta el colon ascendente como normal.

A finales de 2010 se practica nuevos exámenes y evaluación por hematología TP 14,1 seg (VN 11 a $13 \mathrm{seg}) \mathrm{TPT}$ : $18,9 \mathrm{seg}$ (VN de 25 a $35 \mathrm{seg}$ ), Bil total: $0,26 \mathrm{mg} / \mathrm{dl}$ (VN 0,2 a $1,0 \mathrm{mg} / \mathrm{dl})$, directa: $0,16 \mathrm{mg} / \mathrm{dl}(\mathrm{VN} 0,1$ a $0,3 \mathrm{mg} / \mathrm{dl})$, ferritina $5,9 \mathrm{ng} / \mathrm{dl}(\mathrm{VN} 4,63 \mathrm{a} 204 \mathrm{ng} / \mathrm{dl}$ ), hierro sérico: 6 $\mathrm{ug} / \mathrm{dl}$ (VN: 25 a $156 \mathrm{ug} / \mathrm{dl}$ ), Hb: 6,6 g/dl (VN 12,1 a 15 femenino), ácido fólico: 6,30 ng/dl (VN 2,7 a $17 \mathrm{ng} / \mathrm{ml})$, Vit. B12: 1053 pg/ml (VN 200 a 900 pg/ml). Se conceptuó que se trata de anemia ferropénica por pérdida gastrointestinal no identificada.

Ya a mediados de 2011 un $\mathrm{CH}$ muestra $\mathrm{Hb}$ de $6,6 \mathrm{Gr} / \mathrm{dl}$ y recibe nueva transfusión de glóbulos rojos. Un TAC de abdomen mostró proceso inflamatorio de asas intestinales 
delgadas dependientes de yeyuno con ganglios y adenomegalias en el meso, se interroga enfermedad de Crohn.

En mayo de 2012 por solicitud de gastroenterología se practicó estudio de cápsula endoscópica encontrando várices de duodeno distal y yeyuno proximal, vellosidades engrosadas en yeyuno de naturaleza por determinar. Se encontraron uncinarias y angiectasias.

En junio de este año, se presenta con nuevo episodio de melenas, tratada con líquidos y transfusiones de glóbulos rojos.

Gastroenterología planteó posibilidad de tratamiento quirúrgico a finales de 2012 pero no fue posible por problemas administrativos realizar junta quirúrgica. Se practicó eco Doppler esplenoportal que resultó normal.

Consulta de nuevo por melenas ( $\mathrm{Hb}: 5,1 \mathrm{Gr} / \mathrm{dl})$. En vista que cede el sangrado se da de nuevo de alta y se medica con hierro, antiparasitario y se envía de nuevo a consulta externa en la primera semana de noviembre de 2013 (figura 2).

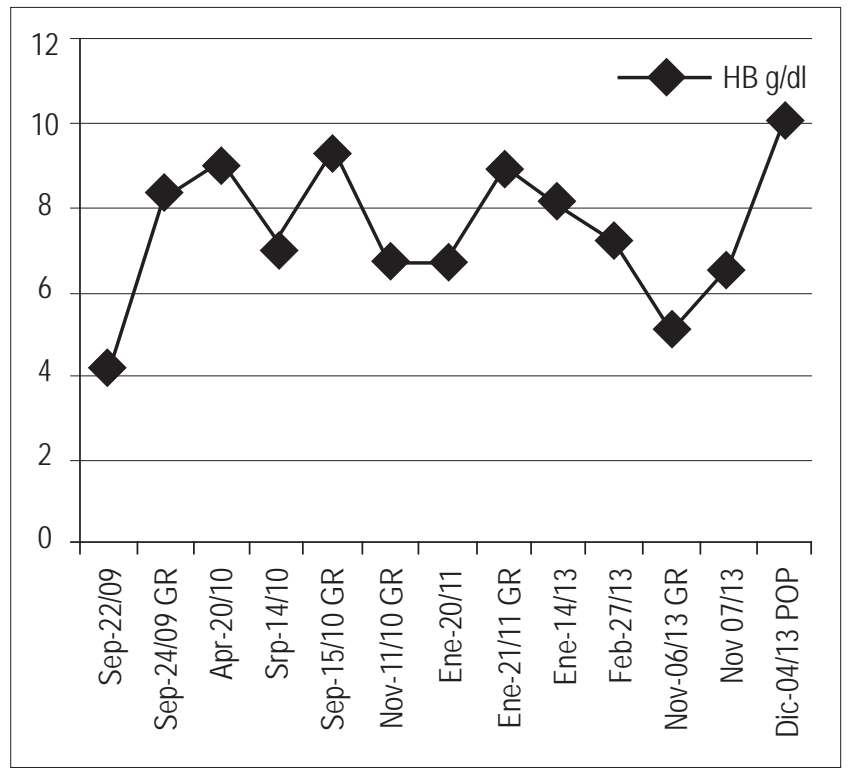

Figura 2. Comportamiento de las hemoglobinas del caso clínico 2009 y diciembre 2013. GR: aplicación de glóbulos rojos.

Reingresa por melenas ( $\mathrm{Hb}$ 6,6 Gr/dl) se examina en conjunto por gastroenterología y cirugía general y se decide hacer laparotomía. Se lleva a cirugía con los siguientes hallazgos: hígado descrito como normal. En yeyuno proximal a $15 \mathrm{~cm}$ del ángulo de Treitz se encontró malformación vascular venosa que compromete toda la pared, en dos segmentos de unos $4 \mathrm{~cm}$ de longitud. Se hace resección del yeyuno comprometido y se reconstruye con anastomosis latero-lateral (Nov-26-2013) (figuras 3 y 4).

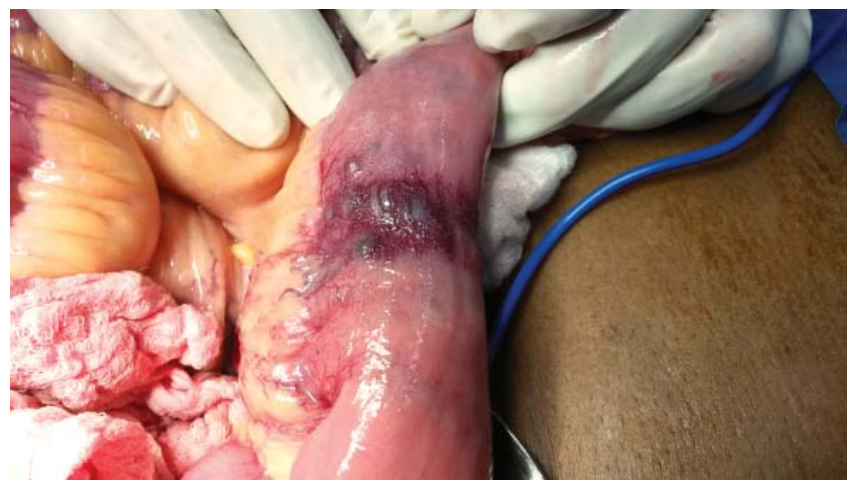

Figura 3. Várices ectópicas (VE) en yeyuno proximal.

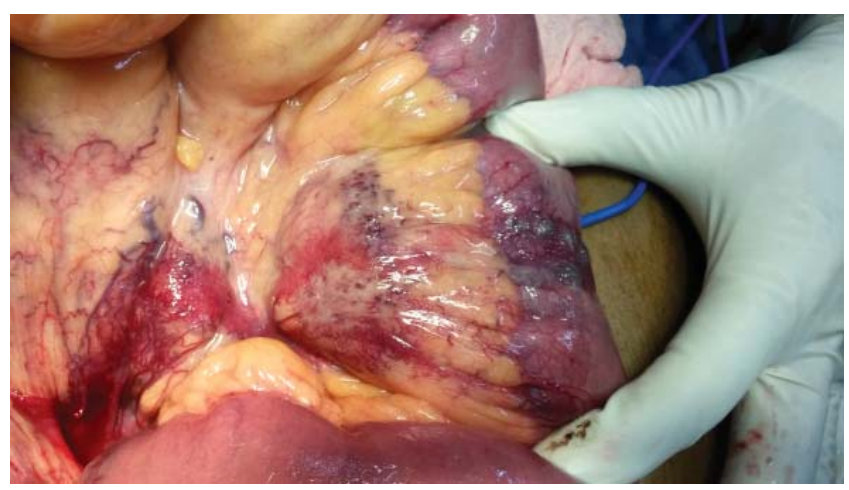

Figura 4. Várices ectópicas VE compromiso peritoneal.

\section{VÁRICES ECTÓPICAS (VE) ASPECTOS FISIOPATOLÓGICOS}

La aparición de dilataciones venosas yeyunales o várices ectópicas (VE) se ha intentado explicar de varias formas:

1. Se ha sugerido que las VE sin presencia de hipertensión portal están relacionadas con cirugías intestinales en las que las anastomosis unan drenajes sistémicos con estructuras con drenajes porta ya que estas anastomosis pueden facilitar su aparición, son ejemplos de ello la pancreatoduodenectomía con resección de afluentes portales, cirugía de colectomía en pacientes con colangitis esclerosante primaria que sufren colitis ulcerativa y desarrollan várices de la ileostomía, también en cirugías en las que se practica bilioenteroanastomosis (17). En los sitios de tejidos de adhesión o estenosis de cirugías intestinales, fístulas arteriovenosas secundarias a trauma también se cree que sean factores que facilitan de aparición de $\operatorname{VE}(27,33,35)$.

El riesgo de desarrollar VE es mayor si hay antecedentes quirúrgicos que involucren o no circulación portal, estenosis de la estoma, o en pacientes con estos ante- 
cedentes quirúrgicos y que desarrollen posteriormente cirrosis e hipertensión portal (17-20, 22, 29, 35). Se ha encontrado que a mayor tamaño de las VE, hay mayor probabilidad de desarrollar el sangrado (16).

2. Si no hay hipertensión portal o antecedentes quirúrgicos se han postulado anomalías congénitas del sistema venoso asociadas como desórdenes hereditarios, hiperplasia nodular linfoide, hepatocarcinoma e idiopáticas $(25-27,34)$.

3. La presencia de VE está descrita en mayor medida en relación con el hecho que el paciente presente hipertensión portal asociada a cirrosis, o trombosis de la vena porta.

Se han denominado várices ectópicas (VE) a colaterales venosas portosistémicas que pueden aparecer en cualquier lugar excepto en la región cardioesofágica (23). Se incluyen en esta denominación várices gástricas aisladas del tipo II (antro cuerpo y alrededor del píloro), las de pared abdominal y espacio peritoneal. Se ha calculado que hasta $8 \%$ de los pacientes con hipertensión portal pueden tener várices ectópicas (17). Las VE se presentan con mayor frecuencia en duodeno seguido de yeyuno íleon, colon, recto, árbol biliar, circulación ovárica y peritoneo. Se han descrito también várices ectópicas en aquellos pacientes con degeneración cavernomatosa o trombosis de la porta $(16,17$, 28, 30-34).

Pueden desarrollarse en cualquier parte de la circulación intestinal o biliar como resultado de cortocircuitos del sistema porta debido a alta presión en este sistema y que esta hipertensión y congestión en la circulación esplácnica alta incrementa la susceptibilidad a la injuria por deterioro de la oxigenación y la isquemia $(16,21,29)$.

De Palma ha definido estos cambios como enteropatía asociada a hipertensión portal y se pueden presentar como lesiones que parecen inflamatorias (eritema, granular, y lesiones friables) y lesiones vasculares (manchas rojas, lesiones que parecen telangiectasias o angiodisplasias y várices). Conceptúa que pacientes con hipertensión portal, con clasificación Child C, grandes várices esofágicas y cambios superficiales en la mucosa del estómago pueden tener $\mathrm{VE}$ con una frecuencia entre 5,5 y $11 \%$ (20).

Se han encontrado VE asociadas a antecedente previo de la ligadura o escleroterapia de várices esofágicas (24).

Para el diagnóstico de han dividido en VE luminales y VE no luminales. Las primeras son aquellas que son fácilmente detectables a la endoscopia y las segundas son las que están en cualquier parte de la cavidad abdominal o la pelvis (16).

Los pacientes con várices duodenales se pueden presentar con hematemesis o un sangrado masivo intestinal bajo. Las várices localizadas distales al duodeno se presentan como melenas o hematoquecia. La várices ectópicas (VE) deberían ser consideradas en todo paciente con hipertensión portal en el que el sangrado no sea localizado por endoscopia alta o baja.

Eventualmente se diagnostican durante la laparotomía en pacientes con abdomen agudo y estado de choque en los que se encuentran VE en zonas como pared intestinal, ligamento falciforme, diafragma, región retrovesical, ligamento esplénico, explicando un sangrado a la cavidad peritoneal que muchas veces puede ser fatal (16).

En otros casos, las VE son diagnosticadas como resultado del estudio de pacientes con anemia crónica, sangrado intestinal manifiesto oculto o que presenten test de sangre oculta positiva (16).

\section{TRATAMIENTO DE LAS VÁRICES ECTÓPICAS (VE)}

Se han encontrado seis tipos de tratamiento para estas várices VE relacionadas o no con hipertensión portal: los endoluminales, los angiográficos por vías convencionales con producción de trombos y los angiográficos con utilización de balones y esclerosantes, los angiográficos vía colaterales, los TIPS y los quirúrgicos.

El tratamiento endoluminal se ha descrito para el control del sangrado las asas de nailon (miniloops) que permiten ligar la várice, y también se han instilado directamente a la VE el cianoacrilato con controles temporales del sangrado $(37,38)$.

Por vía angiográfica se han canalizado venas que drenan las áreas correspondiente a la vena ectópica (VE) y se han pasado primero balones que ocluyen el drenaje y cuando esto se ha conseguido, se instila previamente haptoglobina para prevenir daño renal y luego el esclerosante (oleato de etanolamina) lográndose primero la trombosis y después la esclerosis de la várice.

Otros métodos angiográficos utilizan la embolización directa con espirales (coil embolization) vía angiografía convencional o utilizando venas dilatadas superficiales a través de las cuales se accede al sistema porta $(35,36)$.

Para aquellos pacientes con VE asociadas a hipertensión portal se encontró una serie de 750 aplicaciones de TIPS en las que 28 de estos fueron aplicados para control de várices ectópicas (VE). De estos 12 eran rectales, 8 de una estoma y 4 fueron duodenales y 4 de otros sitios no informados. Se sometieron a TIPS debido al fracaso de terapias endoluminales para el control del sangrado. El control del sangrado luego de aplicación de TIPS se estableció en $60 \%$ de los casos, con un porcentaje de resangrado de $22 \%$ en algunos casos debido a disfunción del shunt. En el resultado influyó marcadamente el estado funcional hepático ya que los Child $\mathrm{C}$ respondieron menos a esta terapéutica (40).

El tratamiento quirúrgico mediante la enterectomía de la zona con las VE también se ha descrito en situaciones en 
las cuales se ha demostrado hipertensión y trombosis de la porta lo que ha dificultado el acceso angiográfico y el resultado reportado ha sido satisfactorio (34).

Se mencionan los tratamientos para VE relacionadas a hipertensión portal porque es con la anormalidad que se ha encontrado más experiencia clínica ya que de aquellas VE sin hipertensión portal solo hay reportes de casos aislados y no hay series como tal para evaluación (39).

\section{DISCUSIÓN}

La presencia de várices ectópicas en intestino proximal (VE) sin estar asociada a hipertensión portal se considera una entidad de rara presentación. Las descripciones relacionadas con esta patología son aisladas y no hay series que permitan conocer su fisiopatología.

Hay alteraciones de la superficie mucosa asociadas a la hipertensión portal por cirrosis diferentes a las comúnmente descritas como las várices esofágicas. La mayor parte de esas alteraciones no son vasculares como lo demuestra un estudio reciente en el que se incluyeron 55 pacientes con cirrosis diagnosticada e hipertensión portal y a los cuales se les practicó estudio de videocápsula endoscópica y se encontró $67 \%$ de anormalidades así: eritema $\mathrm{n}=32(53 \%)$, erosión $\mathrm{n}=10(17 \%)$, angiectasia $\mathrm{n}=9(15 \%)$, várices $\mathrm{n}=$ $4(7 \%)$ y edema velloso $n=4(7 \%)$. La mayor parte de las lesiones fueron localizadas en el yeyuno proximal y medio. La asociación más importante encontrada entre estas lesiones y el examen físico fue la presencia de ascitis (21).

Se identificaron (VE) várices en $7 \%$, que si bien es bajo habrá que tener en cuenta este diagnóstico en pacientes con cirrosis en los cuales no se pueda atribuir persistencia del sangrado a las várices esofágicas.

En una revisión reciente en PUB Med se encontraron 57 casos de VE de intestino delgado entre 1951 y 2003 con un promedio de edad de los afectados de 52,9 años y una asociación con cirugía previa abdominal de $84,2 \%$. La enterectomía fue el tratamiento en 30 de 57 (52\%), de estos 30 casos operados 26 tenían hipertensión portal 26/30 (86\%) y no está descrito el tratamiento para el resto de casos (34).

Shiv y Kumar mencionan una serie de 1.128 casos en las cuales se encontró VE en yeyuno o íleon en 4,6\% en pacientes con hipertensión portal (30).

Con base en los datos previos algunos autores recomiendan sospechar la presencia de VE cuando se presente el cuadro de hematoquecia sin hematemesis e historia de cirugía previa abdominal en paciente con hipertensión portal (36).

Las VE sin hipertensión portal como el caso que describimos es aún menos frecuente y son pocos los reportes en la literatura a este respecto. Se atribuyen las VE sin hipertensión portal a desórdenes vasculares heredables o aun idiopáticos (33).
Las técnicas en desarrollo como la cápsula endoscópica y la enteroscopia podrán identificar con mayor frecuencia estas lesiones. En este caso se reconocieron las várices con la cápsula endoscópica (figuras 5, 6 y 7).

Debido a la muy baja frecuencia de estas lesiones tanto si hay hipertensión portal como si no está presente, hace que el médico no las tenga en consideración fácilmente dentro del diagnóstico clínico diferencial.

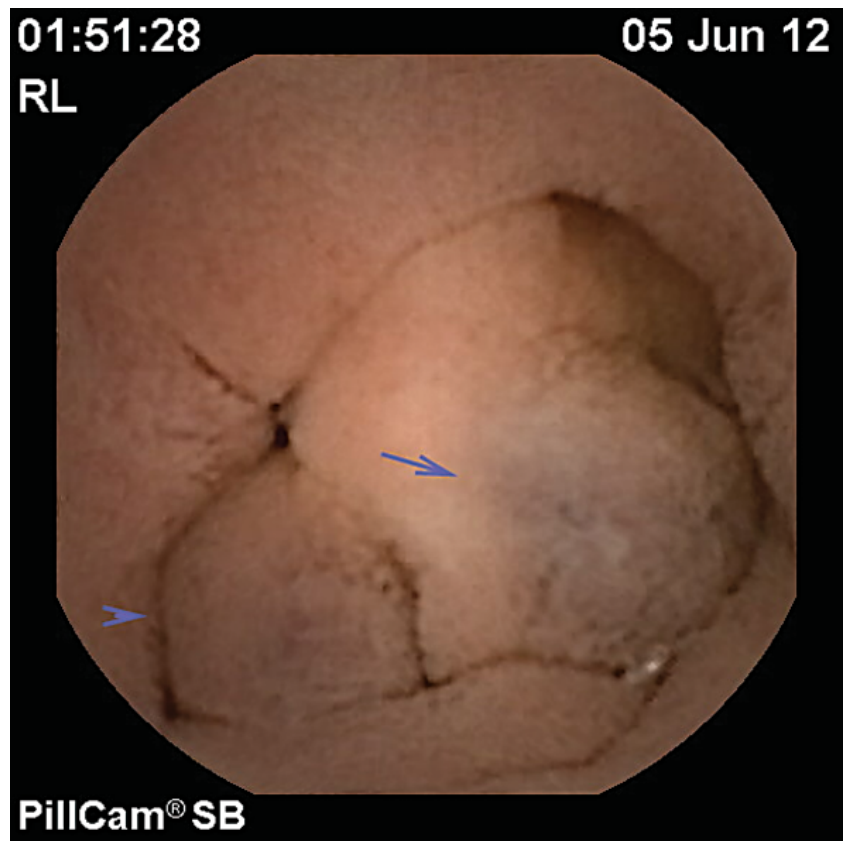

Figura 5. Imágenes de cápsula endoscópica 1.

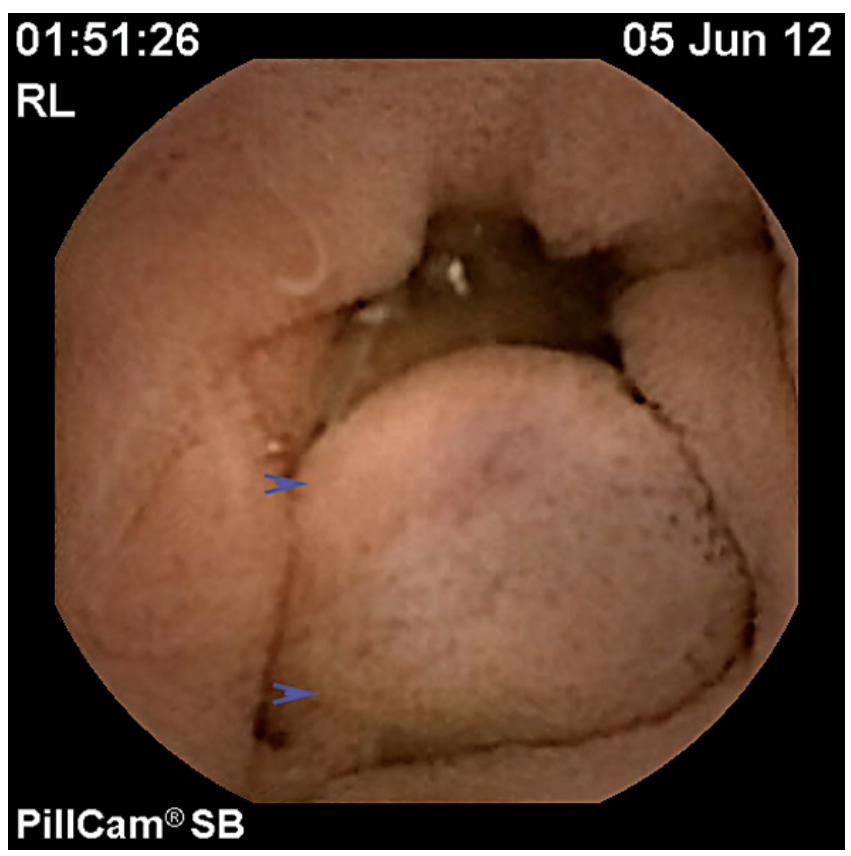

Figura 6. Imágenes de cápsula endoscópica 2. 


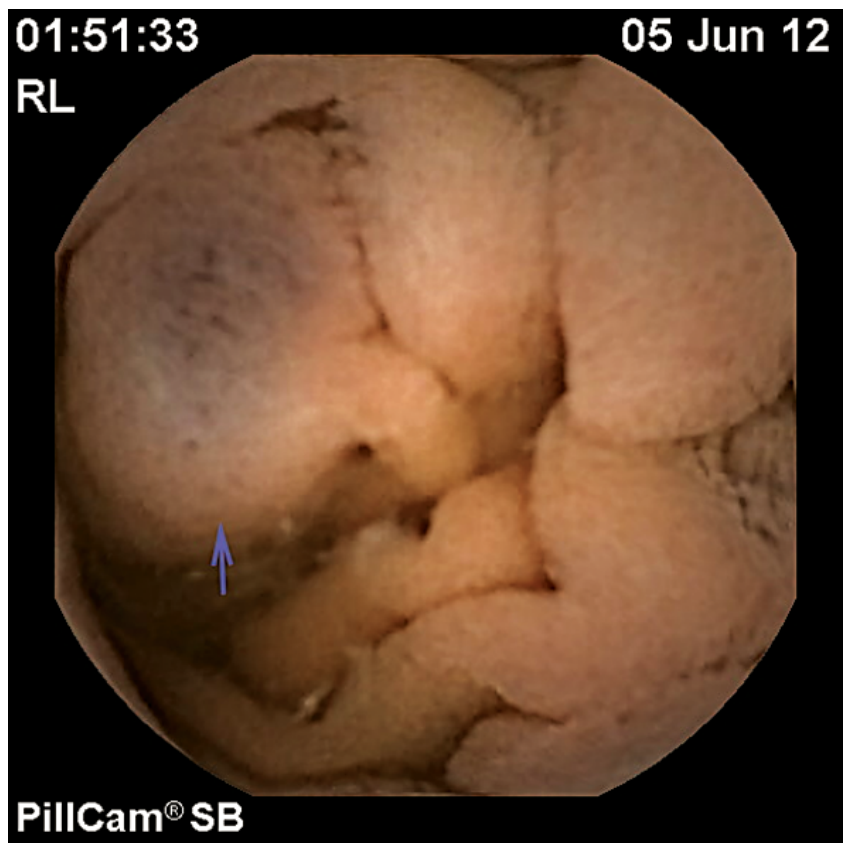

Figura 7. Imágenes de cápsula endoscópica 3.

\section{CONCLUSIONES}

1. Las VE sin hipertensión portal son causas de sangrado consideradas como raras y sin una etiopatogenia satisfactoria.

2. La relación $\mathrm{BUN} /$ creatinina $>33$ puede ser útil para sospechar la localización del sangrado digestivo oscuro.

3. El sangrado digestivo alto en pacientes con hipertensión portal puede ser causado por várices ectópicas (VE).

4. Se puede sospechar sangrado por VE si hay melenas sin hematemesis en pacientes con historia de hipertensión portal $\mathrm{y} / \mathrm{o}$ antecedente quirúrgico abdominal.

5. Los nuevos métodos en desarrollo como la videocápsula endoscópica y la enteroscopia son importantes en el diagnóstico de las VE.

6. Los métodos terapéuticos endoscópicos son parecidos al tratamiento de várices esofágicas pero difieren en cuanto a tamaño, localización de las lesiones (muy variable y menos de 5\% en duodeno y yeyuno proximal), necesidad de tener experiencia en la instrumentación de estas lesiones, lo cual hace difícil predecir el resultado. Los métodos terapéuticos radiológicos requieren personal altamente entrenado.

7. En nuestro medio, la opción quirúrgica es una alternativa viable que debe ser analizada a fondo por los clínicos y cirujanos.

\section{REFERENCIAS}

1. Raju G, Gerson L, Lewis B, American Gastroenterological Association (AGA) Institute Technical. Review on obscure gastrointestinal bleeding. Gastroenterology 2007; 133: 1697-1717.

2. Rondonotti E, Villa F, Mulder C, Maarten J, de Franchis R, Small Bowel capsule endoscopy in 2007 Indications, risk an limitations. World J Gastroenterol 2007; 13 (46):61406149.

3. Farrel J, Friedman L, Review article: The management of lower gastrointestinal bleeding. Aliment Pharmacol Ther 2005; 21: 1281-1298.

4. ASGE. The role of endoscopy in the management of obscure GI bleeding Gastrointestinal Endosc 2010; 72(3): 471-479.

5. Barnert J, Messman H. Diagnosis and management of lower gastrointestinal bleeding. Nat Rev Gastroenterol and Hepatol 2009; 6: 637-646.

6. Mitchell S, Schaefer C. A New View of Occult and obscure gastrointestinal bleeding. Am Fam Physician 2004; 69: 975881.

7. Rockey D. Gastrointestinal Bleeding. En Sleisenger and Fordtrand's. Gastrointestinal and liver disease. $8^{\text {th }}$ edition. Saunders: Elsevier; 2006. p. 255-299.

8. Thomas O, Kovacs G, Jensen D. Lower gastrointestinal bleeding. En Hawkey C, Bosh J, Richter J, Garcia-Tasao G. Text book of Clinical Gastroenterology and Hepatology. Wiley Black Well; 2012. p. 132-138.

9. Lisa L, Lower GI. Bleeding: Epidemiology and Diagnosis. Gastroenterol Clin N Am 2005; 34: 643-664

10. Chalasani N, Clark W, Wilcox CM. Blood Urea Nitrogen to creatinine concentration in gastrointestinal bleeding: A reappraisal. Am J Gastroenterol 1997; 92: 1796.

11. May A, Nachbar L, Schneider M, Christian E. Prospective comparison of push enteroscopy and push- and -pull enteroscopy in patients with suspected small-Bowel bleeding. Am J Gastroenterol 2006; 101: 2016-2024.

12. Arakawa D, Ohmiya N, Nakamura M, Honda W, Shirai O, Itoh A, Hirooka Y, Niwa Y, Maeda O, Ando T. Outcome after enteroscopy for patients with obscure GI bleeding: diagnostic comparison between double-balloon endoscopy and videocapsule endoscopy. Gastrointest Endosc 2009; 69; 4: 866-74.

13. Monsanto P, Almeida N, Lerias C, Figueredo P, Gouveia H, Sofia C. Is there still a role for Intraoperative enteroscopy in patient with obscure gastrointestinal bleeding? Rev Esp Enferm Dig 2012; 104: 190-6.

14. Shiro O, Aoyama T, Imagawa H, Shishido T, Kasuaki C. Effectiveness of polaprezinc for low-dose aspirin-induced small-bowel mucosal injuries as evaluated by capsule endoscopy: a pilot randomized controlled study. Gastroenterology 2013; 13: 108.

15. Galiano M, Cepeda R, García F. Angiodisplasias de intestino delgado, presentación de dos casos. Rev Colomb Gastroenterol 2004; 19: 269-276. 
16. Ahmed H, Khalid Al, Kahtani Al, Fadda M. Updates in the pathogenesis, diagnosis and management of ectopic varices. Hepatol Int 2008; 2: 322-334.

17. Brechman T, Wolf S, Volkmar N, Markus R. Gastrointestinal bleeding 30 years after complicated cholecitectomy. World J Gastroenterol 2010; 16(37): 4747-4750.

18. Saeki Y, Ide K, Kakisawa H, Ishikawa M, Tashiro H, Ohda H. Controlling the bleeding of jejunal varices formed at the site of choledochojejunostomy: report of two cases and a review of literature. Surg Today 2013; 43 (5): 550-5.

19. Watanabe N, Toyonaga A, Seichiro K, Takashimizu S, Kazuhiko O, Kokubo S, et al. Current status of ectopic varices in Japan: Results of a survey by the Japan Society for Portal Hypertension. Hepatol Res 2010; 40: 763-776.

20. De Palma G, Rega M, Masone S, Persico F, Siciliano S, Patrone F, Matantuono L, Persico G. Mucosal abnormalities of the small bowel in patients with cirrhosis and portal hypertension: a capsule endoscopy study. Gastrointest Endosc 2005; 4: 529-34.

21. Aoyama T, Oka S, Aikata H, Nakano M, Watari I, Neshiro N, et al. Small Bowel abnormalities in patients with compensated liver cirrhosis. Dig Dis Sci 2013; 58: 1390-1396.

22. Sandeep K, Dennis A. Occult massive Hemorrhage in a patient with cirrhosis. Ann Am Thorac Soc 2013; 10: 160162.

23. Woong C, Kim S, Woong H, Yun L, Sung J, Sup H, Kim A, et al. A case of variceal bleeding from jejunum in liver cirrhosis. Clin and Mol Hepatol 2013; 19: 78-81.

24. Ohtani T, Kajiwara E, Suzuki N, Kawasaki A, Sadoshima S, Sakata H, Sasaguri Y, Onoyama K. Ileal varices associated with recurrent bleeding in a patient with liver Cirrhosis. J Gastrenterol 1999; 34(2): 264-8.

25. Yamada A, Watanabe H, Shuntaro O, Sugimoto T, Kondo S, Ohta M, Togo G. Surveillance of small intestinal abnormalities in patients with hepatocelular carcinoma: A prospective capsule endoscopy study. Digest Endosc 2011; 23: 124-129.

26. Kastanakis M, Anyfantakis D, Katsougris N, Bobulakis E, Masive gastrointestinal bleeding due to isolated jejunal varices in a patient without portal hypertension. Int J Surg 2013; 4(5): 439-441

27. Bhagwat S, Borwankar S, Ramadwar R, Naik A, Gajaree E. Isolated jejunal varices. J Posgrad Med 1995; 41: 43-4.
28. Joo Y, Kim S, Choi S, Rew J, Kim H. Massive gastrointestinal bleeding from jejunal varices. J Gastroenterol 2000; 35(10): 775-8.

29. Ambiru S, Nakamura S, Mandai Y, Sato T, Kuwahara T, Yokosuka O. Ectopic ileal varices associated with recurrent bleeding: report of case. Surg Today 2011; 41(3): 448-52.

30. Shiv S, Chandan K, Kumar N. Ectopic varices. Clin Liv Dis 2012; 5: 167-172.

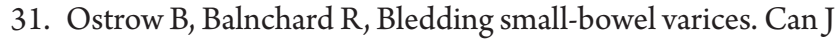
Surg 1984; 27(1): 88-9

32. Yuki N, Kubo M, Kasahara A, Hayashi N, Ito T, Kamada T. Jejunal varices as cause of massive gastrointestinal bleeding. Am J Gastroenterol 1992; 87(4): 514-7.

33. Naofumi E, Shigeaki A, Masao H, Takajashi M, Masatoshi T, Atsushi T. Jejunal varices as cause of massive Gastrointestinal bleeding a case report. Kur Med Journal 1998; 45 (2): 227 230.

34. Ueda J, Yoshida H, Mamada Y, Mizuguchi Y, Shimizu T, Matzumoto S, Ishikawa Y, Kakinuma D, et al. Successful emergency enterectomy for bleeding ileal varices in a patient with liver cirrhosis. J Niponn Med Sch 2006; 73(4): 221-5.

35. Koo S, Soung J, Jae J, Tae L, Seong R, Kim H, Kim J, Kim Y. Jejunal Variceal Bleeding Successfully treated with percutaneous coil embolization. J Korean Med Sci 2012; 27: 321-324.

36. Lee-Guam L, Yin-Mei L, Lenny T, Chang S, Sen-Gee L. Percutaneos paraumbilical embolization as an unconventional and successful treatment for bleeding jejunal varices. World J Gastroenterol 2009; 15(30): 3823-3826.

37. Ghindrim G, Mishim I, Zastavnitsky G. Ruptured duodenal varices successfully treated by mini-loop Ligation: report a case. Chirurgia 2010; 104: 625-629.

38. Nakata M, Nakata W, Isoda N, Yoshizawa M, Sugimoto H, Percutaneous retrograde sclerotherapy for refractory bleeding of jejunal varices: direct injection via superficial epigastric vein. Cadivasc Intervent Radiol 2012; 35: 203-6.

39. Koushi H, Kunihiro Y, Toshio D, Takahashi T, Sato M. Ruptured duodenal varices Successfully treated with Balloon-Occluded Retrograde Transvenous Obliteration: Usefulness of Microcatheters. ARJ 2003; 181: 725-727.

40. Kochar N, Tripathi D, Mcavoy C, Ireland D, Redhead N, Hayes P. Bleeding ectopic varices in cirrhosis: role of transjugular portosistemic stent shunts. Aliment Pharmacol Ther 2008; 28: 294-303. 